\title{
Attenuation Effects of MR Headphones During Brain PET/MR Studies
}

\author{
Aaron Ferguson $\mathrm{CNMT}^{1}$, Jonathan McConathy ${ }^{2}, \mathrm{Yi} \mathrm{Su}^{2}$, Debra Hewing ${ }^{1}$, and Richard Laforest ${ }^{2}$ \\ ${ }^{1}$ Department of Medical Imaging and Radiation Therapeutics, Saint Louis University, St. Louis, Missouri; and ${ }^{2}$ Department of \\ Radiology, Washington University School of Medicine, St. Louis, Missouri
}

\begin{abstract}
PET/MR offers potential advantages over PET/CT that are currently under investigation. One of the challenges of PET/MR is attenuation correction, as there is no simple correlation between MR signal intensity and the attenuation of $511-\mathrm{keV}$ photons detected in PET. Currently, dedicated MR sequences are used
\end{abstract} to segment voxels into categories that are then assigned a predetermined attenuation coefficient. MR hardware such as the imaging table, coils, and headphones are also sources of attenuation. The purpose of this study was to evaluate the effect of MR-compatible headphones on average activity concentration measured with PET/MR. We also present a practical approach to correct for the attenuation effect of headphones using a CTderived attenuation map. Methods: Phantom studies were performed using a 3-L cylindric phantom containing $55 \mathrm{MBq}$ of ${ }^{18} \mathrm{~F}-\mathrm{FDG}$ and water. Images were acquired on a PET/MR device in 2 settings - one with the PET/MR headphones on and one with the headphones off. Phantom images were analyzed to compare activity concentration with headphones on and off. A high-resolution CT and ${ }^{57} \mathrm{Co}$ transmission scan was obtained to construct a PET attenuation map of the headphones. The resulting attenuation map was registered to the phantom data to evaluate the ability to correct for headphone attenuation. One human subject was scanned to evaluate the clinical impact of headphone attenuation and the accuracy of the proposed correction. Results: Activity concentrations measured in the phantom were reduced by as much as $13.2 \%$ with headphones on compared with headphones off. Using the modified attenuation maps that account for attenuation from the headphones resulted in a decrease in the headphone attenuation effect from a maximum of $13.2 \%$ to $1.9 \%$. Comparable attenuation effects were observed in the human brain and were similarly reduced with correction using the modified attenuation maps. Conclusion: MR-safe headphones were a source of attenuation on our PET/MR phantom and human studies. Attenuation effects of headphones should be considered and can be corrected during quantitative brain PET/MR studies.

\footnotetext{
Received Oct. 4, 2013; revision accepted Dec. 23, 2013. For correspondence or reprints contact either of the following:

Richard Laforest, Washington University School of Medicine, Division of Radiological Sciences, Campus Box 8225, 510 South Kingshighway Blvd., St. Louis, MO 63110.

E-mail: laforestr@mir.wustl.edu

Aaron Ferguson, CNMT, Department of Medical Imaging and Radiation

Therapeutics, Saint Louis University, 3437 Caroline St., St. Louis, MO 63104.

E-mail: aaroneferguson@yahoo.com

Published online Feb. 20, 2014.

COPYRIGHT (c) 2014 by the Society of Nuclear Medicine and Molecular Imaging, Inc.
}

Key Words: attenuation; headphones; PET/MR; attenuation correction

J Nucl Med Technol 2014; 42:93-100

DOI: 10.2967/jnmt.113.131995

$\mathbf{H}_{3}$ ybrid imaging modalities provide complementary information to aid in the diagnosis and staging of disease and are powerful tools for research. In PET/CT, the PET provides molecular and functional information whereas the $\mathrm{CT}$ provides corresponding anatomic images and data for attenuation correction. Simultaneous and sequential PET/MR systems have recently been introduced and may offer several advantages over PET/CT. MR imaging provides superior softtissue contrast even in the absence of intravenous MR contrast agents, and multiple MR sequences can be acquired to better clarify tissue and lesion characteristics in a single imaging session. The 3-Tesla Biograph mMR (Siemens Healthcare) allows simultaneous acquisition of MR imaging sequences and PET data at each bed position. This acquisition differs from current PET/CT systems in which the sequentially acquired CT is followed immediately by the PET, allowing a small time difference that can result in image misregistration. Although PET and MR images can be acquired separately and fused using software methods, this arrangement can also lead to image misregistration and is not ideal when using both PET and MR imaging to study rapidly changing biologic processes. MR imaging does not use ionizing radiation; therefore, PET/MR offers the additional benefit of lower radiation dosimetry than the industry standard, PET/CT.

Combining PET and MR in the same gantry was made possible with avalanche photodiodes in lieu of the traditional photomultiplier tubes used in PET. The small size and insensitivity to a magnetic field allowed placement of the PET detector inside the constrained space of the MR imaging scanner. Despite this technical advancement, important questions remain, including the accuracy of MR-based attenuation correction for quantitative PET studies. In PET/CT, attenuation is corrected by converting the Hounsfield units obtained in the CT scan to the corresponding 511-keV equivalent attenuation coefficient. Because of the similarity of the physical interaction of $\mathrm{x}$-rays and $511-\mathrm{keV}$ photons, a scaling 
approach can be used (1). MR signal is based on the density and magnetic relaxation of protons rather than photon attenuation; therefore, there is no direct relationship between MR intensity and attenuation maps for PET. For example, both cortical bone with relatively high photon attenuation and air with low photon attenuation have comparatively low signal on most MR sequences. Thus, MR-based attenuation correction must be approached differently from CT-based attenuation correction.

One approach to MR attenuation correction is the 2-point Dixon sequence, which is approved by the Food and Drug Administration $(2,3)$. The Dixon sequence is a spin echo that uses 2 echo times, when fat and water are in and out of phase. Tissues with fat and water can then be separated. The Dixon sequence segments tissues into 4 classifications (background, lungs, fat, or soft tissue) (3). Voxels are then assigned an attenuation coefficient based on their tissue classification to create an MR-based attenuation map for the PET data. Cortical bone has a low mobile proton density with short magnetic relaxation times and therefore is not recognized accurately on currently implemented clinical sequences. With the Dixon sequence, cortical bone is misclassified as soft tissue or fat, thus underestimating its attenuation effects. This segmentation error can lead to errors in standardized uptake value on the order of $15 \%$ for regions within cortical bone (3). Similarly, the material composing the MR headphones has a low mobile proton density and short magnetic relaxation times. Dixon sequences have been shown to work well for attenuation correction in the body but may not be appropriate for quantitative studies, particularly in the head, neck, and pelvis, where there is a relatively higher ratio of bone to soft tissue (3). The Dixon sequence also will likely be problematic for several patient-positioning devices such as headphones, which are not visualized with MR imaging.

Ultrashort echo time (UTE) MR sequences have been proposed that use a greatly reduced echo time to capture rapidly decaying signals and have potential for attenuation correction purposes in PET/MR data. The UTE sequence has demonstrated the ability to detect a signal from cortical bone (4) and, thus, might be used to help define the proper attenuation for cortical bone (5). Although the UTE sequence shows promise, it is still a work in progress for clinical use (6). A combined UTE-Dixon sequence has been recently proposed that may be a more comprehensive approach to MR attenuation correction in the future (7). The UTE-based sequences are not yet approved by the Food and Drug Administration for clinical use.

In addition to correcting for tissue in the body, the system must also correct for hardware attenuation. Fixed attenuating material outside the patient includes the imaging table and head coil. The imaging table is more complex in PET/MR than in PET/CT, as it also contains the MR spine coils composed of solid plastic, wires, and electronic components. Nonfixed equipment includes surface coils, electrocardiography devices, headphones, and positioning aids. The system can correct for fixed equipment with preloaded attenuation maps; however, nonfixed equipment must be dealt with separately. Positioning aids have demonstrated errors in activity concentration calculations and introduced artifacts on PET images (8). MR surface coils have also been shown to be a source of attenuation, and a technique for their attenuation compensation has been recently presented (9-13). Although the attenuation effects of MR coils and positioning aids have been studied, there remains a need to measure the magnitude and correct the attenuation effects of MR-compatible headphones for PET/MR studies. Delso et al. evaluated the attenuation properties of MR-compatible headphones without proposing a correction method (9).

Acoustic noise during MR studies can cause discomfort and potentially hearing damage; therefore, hearing protection is required. Hearing protection can be provided using sounddampening earplugs or MR-safe pneumatic headphones (Fig. 1A). The headphones also allow technologists to communicate with the patient during scans. Communication may be necessary to provide instructions during functional studies or breath-hold sequences. The patient may also listen to music through the headphones, providing entertainment during long procedures. MR-safe pneumatic headphones are usually constructed of polyamide and glass fiber and some plastic foam padding ( 6 ). These materials allow the headphones to

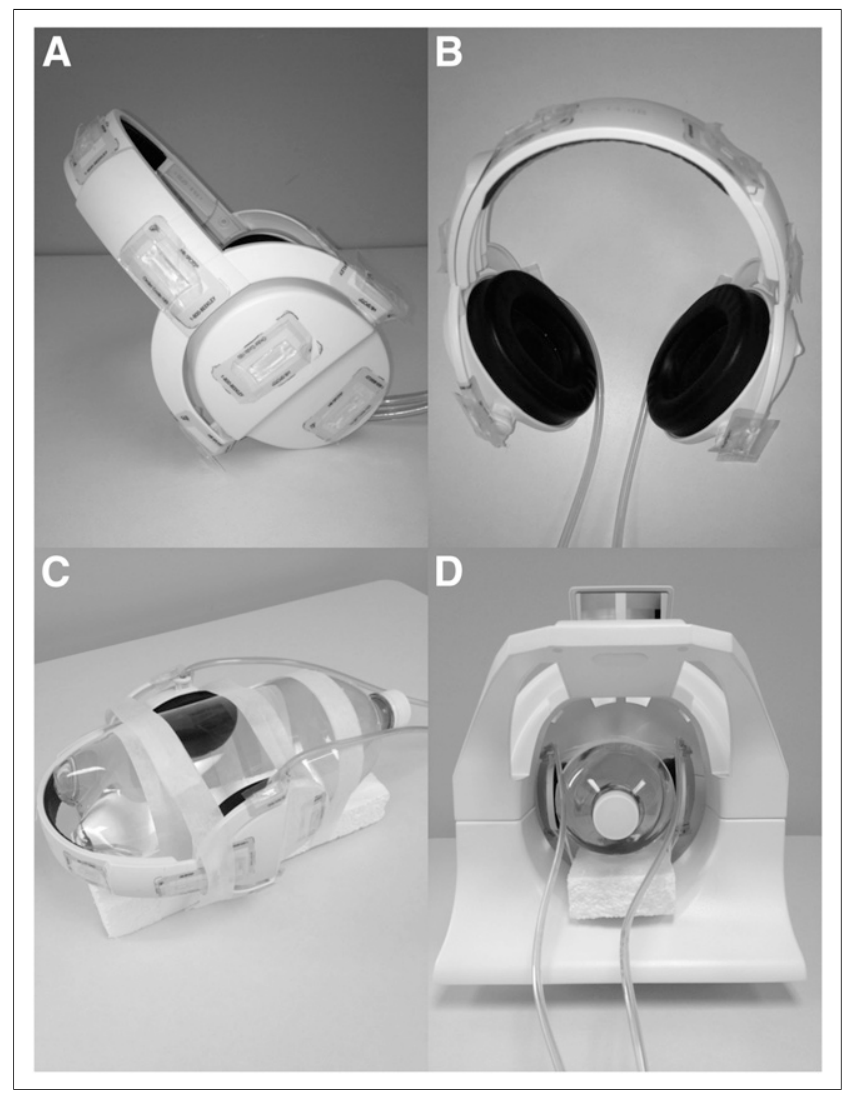

FIGURE 1. (A and B) Side view of headphones showing 12 fiducial markers $(A)$ and top view of headphones with fiducials (B). (C and D) Headphones secured to Styrofoam (C) and placed in head coil (D). 
go undetected during MR studies with current clinical sequences. However, they still have the potential to attenuate the 511-keV PET photons and affect the quantitative accuracy of PET images of the head. Because MR-safe headphones are not visualized by clinical MR sequences, MR-based attenuation correction cannot be readily performed for the headphones. Thus, in this study, we evaluated the attenuation effect that MR-compatible pneumatic headphones have on PET images of the head. We also demonstrate the feasibility of incorporating a CT-based headphone attenuation map of the headphones into the PET/MR attenuation algorithm using bimodal fiducial markers.

\section{MATERIALS AND METHODS}

\section{Phantom Acquisitions}

Radioactive Phantom Without and With Headphones. A 3-L plastic bottle was completely filled with water and $55.3 \mathrm{MBq}$ of ${ }^{18}$ F-FDG. The bottle was secured on a block of Styrofoam (The Dow Chemical Co.) with paper tape, placed in the PET/MR head coil (Fig. 1B), and scanned on a Biograph mMR with the 2-point Dixon MR imaging sequence and a simultaneous 5-min PET emission acquisition (parameters listed in Table 1).

Twelve $22 \times 8 \times 2 \mathrm{~mm}$ Radiance filled packet self-adhesive disposable MR imaging skin markers (Beekley Corp.) were placed on the headphones as fiducial markers for visualization in the PET/MR images. Four fiducials were placed on each earpiece, with 2 fiducial markers placed on the outer shell and 2 on the hinges where the earpieces pivot with respect to the strap (Fig. 1A). The 4 remaining fiducials were placed on the thickest portion of the strap (Fig. 1A). The headphones with fiducials were attached to the bottle, secured with paper tape, and placed back in the head coil (Fig. 1B). The PET/MR imaging was repeated using the same acquisition parameters. A UTE sequence (details in Table 1) was acquired during the headphoneson acquisition to evaluate its ability to localize the headphones. The
Dixon and UTE attenuation sequences were completed during the PET acquisition. The 2-point Dixon sequence produced in-phase, out-of-phase, water, and fat images. These images were used to produce a segmented image scaled to proper attenuation for water and fat at $511 \mathrm{keV}$ suitable for PET image reconstruction.

High-Resolution CT. The headphones with the 12 fiducial markers were attached to an empty 3-L plastic bottle. A 512-mm CT scout scan was acquired on a Biograph 40 TruePoint PET/CT scanner (Siemens Healthcare) followed by a high-resolution, low-amperage CT scan with the following parameters: $120-\mathrm{kV}$ collimation, $1.5-\mathrm{mm}$ slice thickness $(40 \times 0.6 \mathrm{~mm}), 35 \mathrm{mAs}$, and $300-\mathrm{cm}$ FOV. Images were reconstructed with $1.5-\mathrm{mm}$ slice thickness and $0.48-\mathrm{mm}$ pixel size on a $512 \times 512$ matrix. This scan provided the template images that were registered to the anatomic attenuation map and were evaluated for their attenuation properties.

Transmission Scan. A 45-min transmission scan of the headphones with fiducial markers was obtained on a Focus 220 smallanimal PET scanner (Siemens Medical Solutions) using a rotating ${ }^{57}$ Co point source. A 2.5 -cm-diameter, $50-\mathrm{mL}$ plastic centrifuge tube was filled with water only and placed between the earpieces. The centrifuge tube containing water was used as an internal reference to verify the accuracy of the transmission-derived attenuation correction map.

Human Brain Scans. PET/MR images of the head were acquired on a single human subject to evaluate the effects of headphone attenuation and our ability to correct the attenuation effect in a human brain. Washington University's Human Research Protection Office approved this study, and the subject gave written informed consent. The subject had been injected with $377.4 \mathrm{MBq}$ of ${ }^{18} \mathrm{~F}-\mathrm{FDG}$ for a previous PET/CT examination and therefore did not receive additional radiation by participating in this study. One acquisition with headphones on including the 12 fiducials placed in the configuration displayed in Figure 1A and one acquisition with headphones off were performed using the parameters listed in Table 1. A high-resolution magnetization-prepared rapid-acquisition gradient

TABLE 1

Acquisition Parameters

\begin{tabular}{|c|c|c|c|}
\hline MR parameters & MRAC Dixon AC & MRAC UTE AC & MPRAGE \\
\hline Acquisition time (min) & $00: 19$ & $01: 40$ & $04: 45$ \\
\hline Voxel size (mm) & $2.6 \times 2.6 \times 3.12$ & $1.6 \times 1.6 \times 1.6$ & $1.1 \times 1.1 \times 1.2$ \\
\hline Orientation & Coronal & Transverse & Sagittal \\
\hline Slices per slab & 128 & 192 & 176 \\
\hline Matrix size & $192 \times 126$ & $192 \times 192$ & $256 \times 256$ \\
\hline Field of view (mm) & 500 & 300 & 270 \\
\hline Repetition time (ms) & 3.60 & 11.94 & 2,100 \\
\hline First and second echo time (ms) & 1.23 and 2.46 & 0.07 and 2.46 & 2.95 \\
\hline Flip angle & $10.0^{\circ}$ & $10.0^{\circ}$ & 9.0 \\
\hline Parallel acquisition technique mode & GRAPPA 2 (Siemens) & None & GRAPPA 2 (Siemens) \\
\hline \multicolumn{4}{|l|}{ PET parameters } \\
\hline Emission scan & $5 \mathrm{~min}$ & & \\
\hline Reconstruction iterations & 3 & & \\
\hline Reconstruction subsets & 21 & & \\
\hline Postreconstruction filter & Gaussian, 4 mm & & \\
\hline Number of slices & 127 & & \\
\hline Matrix & $256 \times 256$ & & \\
\hline Orientation & Transverse & & \\
\hline Voxel size (mm) & $1.4 \times 1.4 \times 2.03$ & & \\
\hline
\end{tabular}

$\mathrm{AC}=$ attenuation correction; GRAPPA = generalized autocalibrating partially parallel acquisition; MRAC = MR-based attenuation correction. 


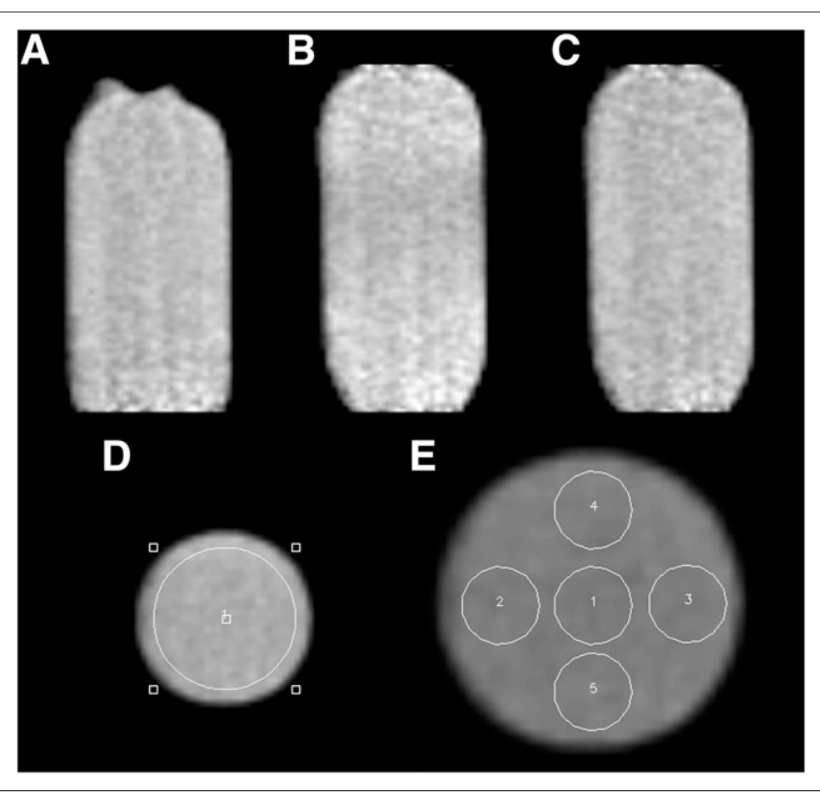

FIGURE 2. (A) Three-liter radioactive phantom without headphones demonstrates uniform distribution of activity. (B) Threeliter radioactive phantom demonstrates decrease in activity in center region with headphones on uncorrected. (C) Three-liter phantom using corrected $\mu$ maps demonstrates uniform activity concentration with headphones on. (D) Large, circular region of interest replicated across all transverse planes. (E) Five small, circular regions of interest replicated across all transverse planes. $1=$ center; 2 = right; 3 = left; $4=$ upper; $5=$ lower.

echo (MPRAGE) sequence was acquired to obtain images with fine anatomic detail. The pneumatic tubing on the headphones was placed anteriorly for all imaging. All MR sequences were performed simultaneously with PET emission acquisitions.

\section{Processing}

PET/MR Data. PET images were reconstructed with 3-dimensional (3D) ordered-subsets expectation maximization using the standard parameters ( 3 iterations, 21 subsets, gaussian postreconstruction filter at $4 \mathrm{~mm}$ ) and the default 2-point Dixon attenuation correction. The reconstructed images were converted to a volumetric 3D dataset and analyzed with ASIPro small-animal PET software (Siemens Medical Solutions). A circular region of interest encompassing all but the edges of the bottle was drawn on a transverse slice of the dataset (Fig. 2D). The region was replicated across all transverse planes acquired. The mean activity concentration in $\mathrm{Bq} / \mathrm{mL}$ was calculated for each slice. The average mean activity concentration of the whole bottle without headphones was compared with the decay-corrected activity concentration of slices of the bottle with headphones. Additional analysis was performed by evaluating 5 smaller individual regions of interest. Five circular ( 8 by 8 pixel) regions were drawn on a transverse slice of the 3 - $\mathrm{L}$ bottle (Fig. 2E). Each region was replicated across all transverse planes acquired. The mean activity concentration of the slices with headphones was compared with the mean activity concentration of the bottle without headphones for the 5 individual regions.

High-Resolution CT. The CT images of the bottle and headphones were processed by manually removing all pixel elements not belonging to the headphones. Regions were drawn using ImageJ software, version 1.47t (National Institutes of Health), extracting everything except the headphones to produce a CT template of the headphones for application to the MR attenuation correction dataset (Figs. 3A and 3B). The spatial location of the fiducial markers with respect to the $\mathrm{CT}$ scanner reference coordinates was then extracted from the 3D volumetric images. The CT templates of the headphones were then separated into 4 parts: right and left sides of the strap and right and left earpieces.

Transmission Scan. The ${ }^{57}$ Co transmission image (attenuation map) of the headphones acquired in the Focus 220 was used to measure the attenuation coefficient of the headphones (Fig. 3C). The reconstructed images from this transmission scan (using the filtered backprojection algorithm with frequency cutoff at Nyquist) were analyzed with ASIPro. A circular region of interest encompassing all but the edges of the water-filled centrifuge tube was drawn on a transverse slice of the dataset (Fig. 3D). The region was replicated across all transverse planes acquired. Twelve headphone regions of interest were also drawn on transverse slices (Fig. 3D).

The spatial location of fiducial markers, with respect to the MR scanner coordinates, was then extracted from the in-phase MR images acquired during the 2-point Dixon acquisition. This sequence provided the clearest visualization of the fiducial markers. A central point on each fiducial was localized in all 3 planes. A 3D rigid transformation of the CT template of the headphones was then performed to minimize the sum of the square difference of the

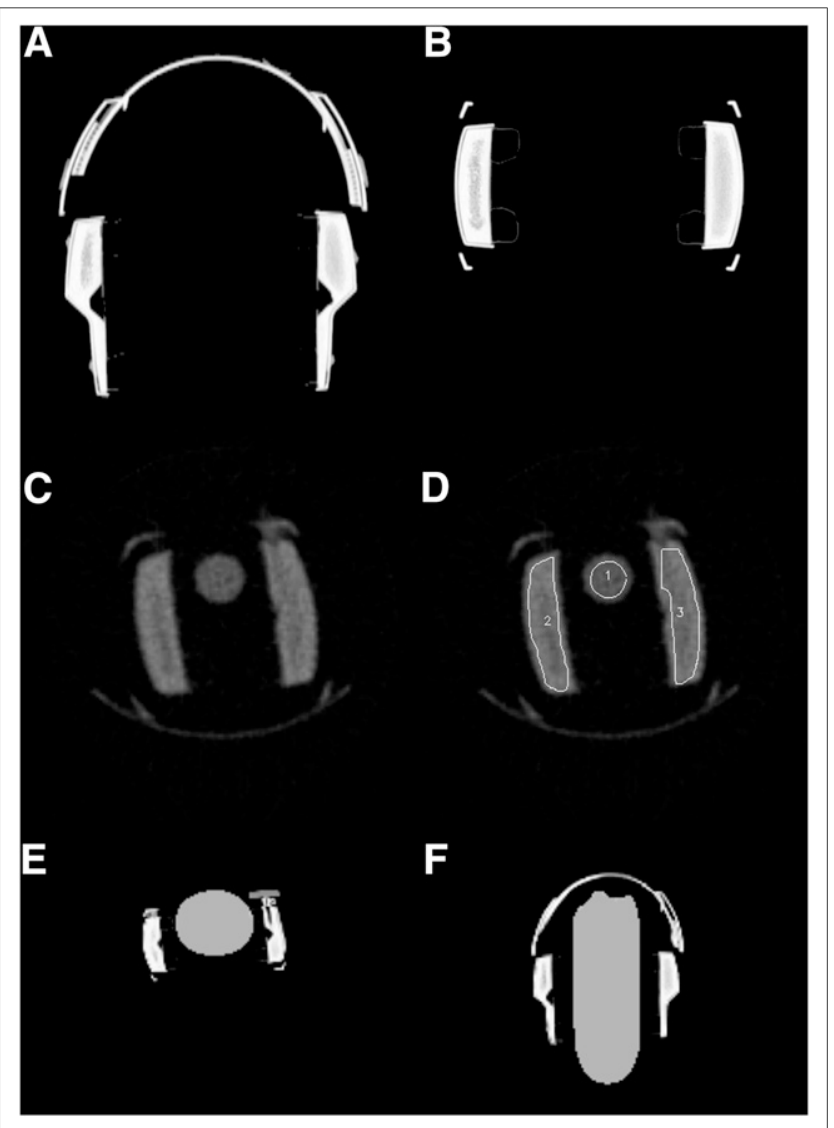

FIGURE 3. (A) View of coronal CT template created from highresolution CT transmission scan. (B) Transaxial CT template. (C) Axial view of ${ }^{57} \mathrm{Co}$ transmission scan. (D) Axial ${ }^{57} \mathrm{Co}$ transmission with representative view of how regions of interest were drawn on headphones and water-filled centrifuge tube. (E) Axial view of modified $\mu$ map. (F) Coronal view of modified $\mu$ map. $1=$ center; 2 = right; 3 = left. 
spatial locations of the fiducial markers as seen on the CT template and in-phase MR images. The headphones are flexible to accommodate the unique size and shape of individual human heads. Each earpiece also pivots, resulting in a changing geometry that makes accurate registration challenging. We divided the headphones into 4 separate pieces to address this problem. Rigid-body registration was performed using the 2 fiducial markers on the strap and the 2 fiducial markers on the hinges for the right side of the strap. For the right earpiece, the 2 fiducial markers on the earpiece and the 2 fiducial markers on the hinges were used. The procedure was repeated for the left strap and earpiece using the corresponding markers. The registered CT images in Hounsfield units were then scaled to the proper PET attenuation coefficient and added to the MR attenuation map to create a modified $\mu$ map (Figs. 3E and 3F). The algorithm was written in IDL, version 8.1 (Interactive Data Language; Exelis Inc.), and used the Powell minimization algorithm.

The headphones-on data reconstruction was repeated with the same parameters, 3D ordered-subsets expectation maximization (3 iterations, 21 subsets, gaussian postreconstruction filter at $4 \mathrm{~mm}$ ) using the corrected attenuation map that included the headphones. The reconstructed images were converted to a volumetric 3D dataset and analyzed with ASIPro. A circular region of interest encompassing all but the edges of the bottle was drawn on a transverse slice of the dataset (Fig. 2D). The region was replicated across all transverse planes acquired. The mean activity concentration in $\mathrm{Bq} / \mathrm{mL}$ was calculated for each slice. The average mean activity concentration of the whole bottle without headphones was compared with the decay-corrected activity concentration of the slices of the bottle with headphones using the modified $\mu$ map. This process was repeated for the 5 individual small, circular regions of interest.

Human Brain Scan. Human PET images were reconstructed with 3D ordered-subsets expectation maximization with the 2-point Dixon attenuation file. Three PET images, that is, without headphones, with headphones and attenuation map, and with headphones and modified attenuation map, were aligned with the simultaneously acquired MPRAGE (parameters listed in Table 1) data during the scan without headphones for quantitative regional analysis. The MPRAGE scan was processed by FreeSurfer, version 5.1 (Martinos Center for Biomedical Imaging), to automatically segment the brain into various anatomic regions. The regional decay-corrected activity concentration of each anatomic region of interest (ROI) as defined by FreeSurfer was determined for all 3 PET datasets. With the scan without headphones used as a reference, relative measurement error was calculated for each region and compared between the reconstruction with and without correction for headphone attenuation. Ratio images of headphones-on over headphones-off and headphones-corrected over headphones-off were created using ASIPro. A digital mask was applied to include counts within the head only.

\section{RESULTS}

\section{CT Template and Transmission Scan}

Figures $3 \mathrm{~A}$ and $3 \mathrm{~B}$ show the coronal and transverse views of the CT template of the headphones from the Biograph 40, and Figure 3C shows transverse transmission attenuation images as measured in the Focus 220. The water regions of interest on the Focus 220 transmission image (Fig. 3D) revealed a measured attenuation coefficient of $0.092 \mathrm{~cm}^{-1}$ (nominal value of $0.096 \mathrm{~cm}^{-1}$ ), indicating an accurate calibration of the Focus 220 for attenuation correction measurement. The average measured attenuation value from the 12 regions of the headphones was $0.121 \mathrm{~cm}^{-1}$. The accuracy of the registration method resulted in an average root mean square difference of less than $1 \mathrm{~mm}$, which is less than the reference MR pixel size. The measured CT Hounsfield units of the headphones (average value, 1,399) were then scaled to the attenuation coefficient as seen on the ${ }^{57} \mathrm{Co}$ transmission measurement.

\section{Phantom Data}

Phantom Uncorrected and Corrected. Figure 2A presents the axial profile of the measured activity concentration along the water- and ${ }^{18}$ F-FDG-filled bottle without headphones. The uniform activity concentration observed along the phantom indicates an appropriate attenuation correction in the absence of headphones. With the headphones in place, a decrease in activity can be visualized in Figure $2 \mathrm{~B}$ that corresponds to the location of the headphones on the bottle. Figure $2 \mathrm{C}$ displays a more uniform activity concentration after correction with the modified $\mu$ map. Figure 4A displays the difference in activity concentration between headphones off, on, and corrected for each slice of the phantom. Figure 4B displays the percentage difference in counts between headphones off versus on and headphones off versus corrected for each slice of the phantom. Activity concentration in the phantom was reduced by as much as $13.2 \%$ with headphones on compared with headphones off. Use of the modified $\mu$ map decreased the headphone attenuation effect from a maximum of $13.2 \%$ to $1.9 \%$ (using slices 20-100) in the headphone region of the phantom.

The average percentage difference in the count statistics for the entire headphone region on the bottle (using slices 20-100) with headphones on compared with off was $6.5 \%$. The average percentage difference in the count statistics for the headphone region on the bottle (using slices 20-100) with headphones on using the corrected $\mu$ map compared with off was $0.6 \%$. Figure $5 \mathrm{~A}$ summarizes these differences.

Figure $4 \mathrm{C}$ displays the difference in activity concentration of the 5 regions between headphones off and on for each slice of the bottle. Figure 4D displays the difference in activity concentration of the 5 regions between headphones off and corrected for each slice of the bottle. Figure 4E displays the percentage difference between headphones on compared with off of the 5 smaller regions of interest. Evaluation of the 5 small, circular regions revealed maximum decreases in activity concentration in the headphone area (slices 20100 ) of $10.9 \%$ in the center region, $14.0 \%$ in the right region, $16.4 \%$ in the left region, $9.7 \%$ in the upper region, and $12.2 \%$ in the lower region. Figure 4F displays the percentage difference in activity concentration using the corrected $\mu$ map in the headphone area (slices 20-100). The corrected maximum percentage differences were $3.9 \%$ in the center region, $4.7 \%$ in the right region, $2.5 \%$ in the left region, $2.1 \%$ in the upper region, and $1.8 \%$ in the lower region. Figure $5 \mathrm{~B}$ summarizes these differences. 


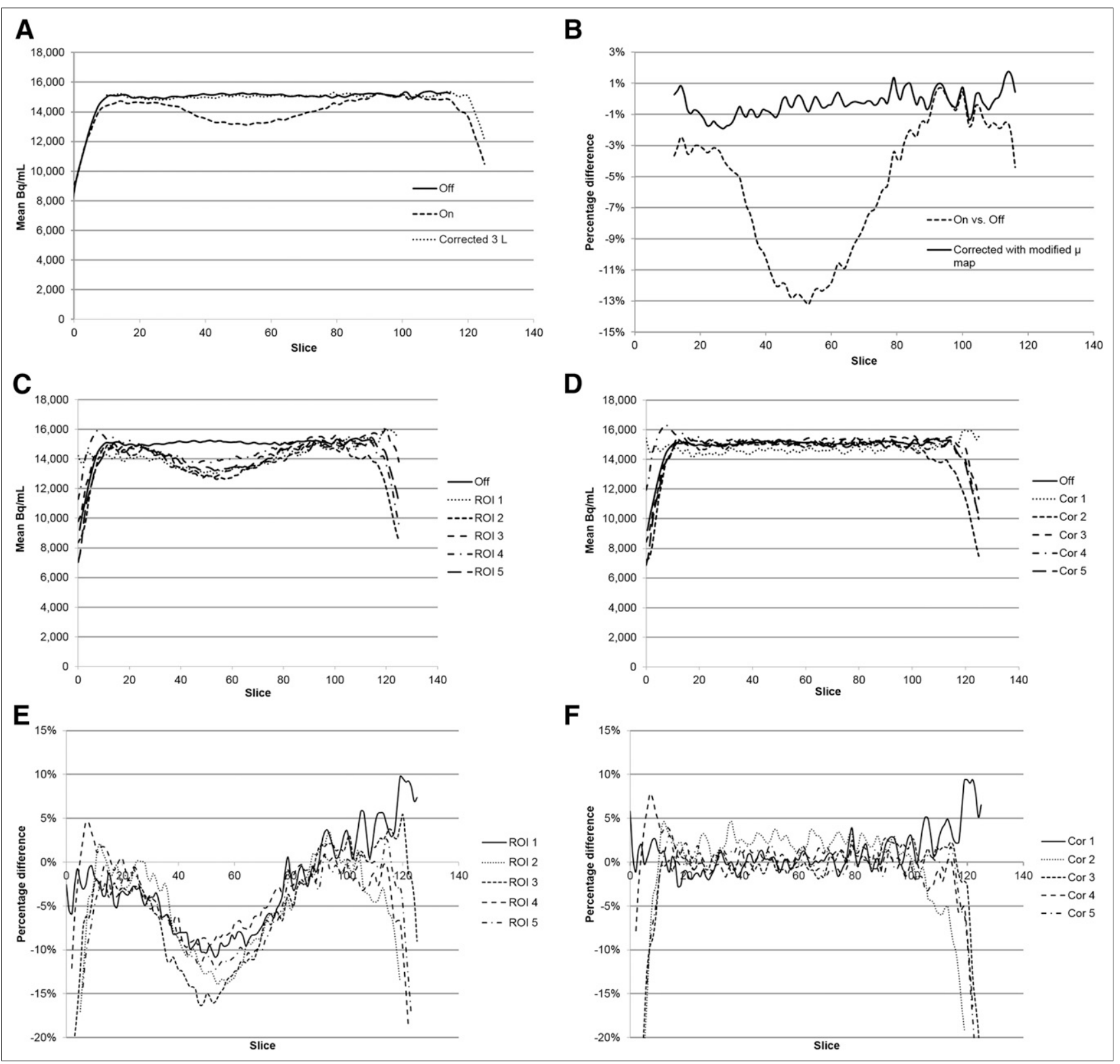

FIGURE 4. (A) Difference in activity concentration between headphones off, on, and corrected for each slice of phantom. (B) Percentage difference in counts between headphones off vs. on and off vs. corrected for each slice of phantom. (C) Difference in activity concentration of the 5 regions between headphones off and on for each slice of bottle. (D) Difference in activity concentration of the 5 regions between headphones off and corrected for each slice of bottle. (E) Percentage difference between headphones on compared with off of the 5 smaller regions of interest. (F) Percentage difference in activity concentration using corrected $\mu$ map in headphone area (slices 20-100). Cor = corrected. A color version of this figure is available as a supplemental file at http://tech.snmjournals.org.

The average decrease in count statistics over the entire headphone area in the small regions (slices 20-100) was $5.6 \%$ in the center region, $6.1 \%$ in the right region, $7.7 \%$ in the left region, $4.6 \%$ in the upper region, and $6.2 \%$ in the lower region. Figure 5B displays the percentage difference between headphones on and off in these regions. Figure 5B also summarizes the percentage difference between headphones off and headphones on using the data reprocessed with the corrected $\mu$ map. The average decrease in count statistics over the entire headphone area in the small regions (slices
20-100) using the corrected $\mu$ map was $1.0 \%$ in the center region, $2.2 \%$ in the right region, $0.9 \%$ in the left region, $0.9 \%$ in the upper region, and $0.9 \%$ in the lower region.

Human Subject Data. Without correction for the attenuation caused by the headphones, substantial underestimation in activity concentration was observed for brain regions near the headphones. Table 2 displays the percentage change in counts of 12 selected regions commonly evaluated for amyloid imaging. The average of the right and left inferior temporal cortex was underestimated by $14.0 \%$, and the average 


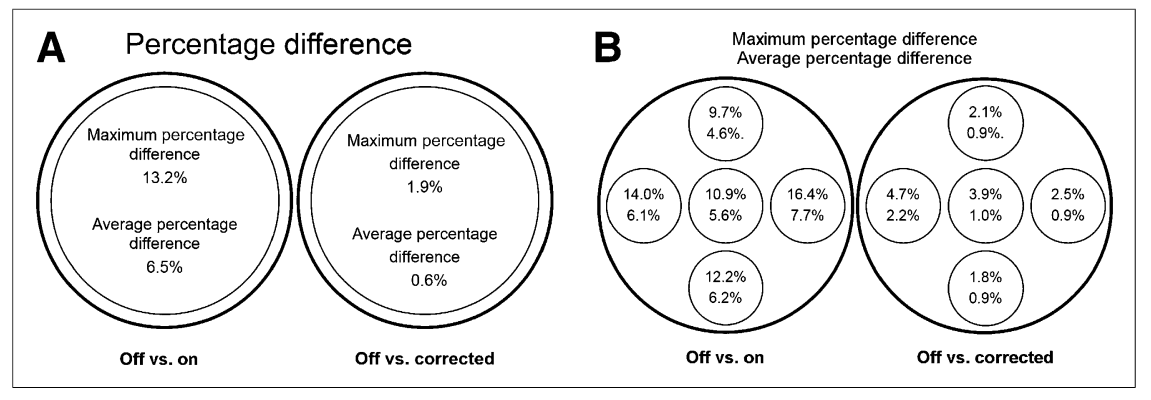

FIGURE 5. Maximum and average percentage difference for off vs. on and off vs. corrected. (A) Large regions of interest (slices 20-100). (B) Small regions of interest (slices 20-100). of the right and left cerebellar cortex was underestimated by $14.4 \%$. After correction for headphone attenuation, these underestimations were reverted. The relative measurement error was a $2.1 \%$ overestimation for the inferior temporal cortex and a $0.2 \%$ overestimation for the cerebellar cortex. Figure 6A displays a coronal image showing the ratio of measured activity concentration from the PET scan acquired with headphones over the scan without headphones. Figure 6B displays a coronal image showing the ratio of measured activity concentration of headphones with attenuation correction over the scan without headphones. A decrease in image intensity is seen in the area between the headphones, which is reverted with correction using the modified $\mu$ maps.

\section{DISCUSSION}

Accurate MR-based attenuation maps are an important challenge for PET/MR. Inaccurate attenuation maps have been shown to cause positive or negative bias in reconstructed images (14). MR-based attenuation correction is particularly challenging for materials that have relatively high attenuation of photons but are not visualized with MR sequences. In this study, we demonstrated that MR-safe pneumatic headphones are a potential source of attenuation during PET/MR examinations of the head and that proper attenuation correction for these devices needs to be used for quantitative PET imaging.

In our phantom study, decreases of up to $13.2 \%$ in activity concentration were observed with headphones on compared with headphones off using Dixon-based MR attenuation correction. With CT-based attenuation correction and registration with CT/MR-visible fiducials, the MR-based attenuation map could be corrected to a $1.9 \%$ decrease with headphones on compared with off. This degree of error is relatively small and is likely to be acceptable for many quantitative PET studies of the brain. This method could potentially be applied to other patient-positioning aids and accessories used during PET/MR studies. Headphone attenuation may not cause problems in qualitative clinical examinations but might be more important in research or quantitative studies.

A larger attenuation effect was seen in the regions closest to the headphones (regions 2 and 3 in Fig. 2D). Paulus et al. observed similar findings with surface coils (12), because of the larger number of lines of response crossing in the vicinity of the headphones. Because the headphones are now included in the attenuation file, a better estimate of the scatter distribution can be calculated and thus a more accurate scatter correction established. The attenuation in these regions decreased from $14 \%$ and $16 \%$ to below 5\% with correction. Correction with modified $\mu$ maps may enable more accurate quantification of metabolic activity in the temporal lobes and cortex.

A disadvantage of the fiducials is that their placement and configuration must remain constant over time. If the fiducials are removed intentionally or accidentally, the CT scan for localization would need to be repeated with the new fiducial configuration. The fiducials will also be visible in all MR images of the head if included in the field of view. Interpreting physicians should be aware that the fiducials are in place and may occasionally be visible on images. A second disadvantage is that localization of the fiducial markers is currently a manual process. Our manual attenuation correction process took about $30 \mathrm{~min}$, including the time required to transfer data to and from the PET/MR workstation. Further work will include a method to automatically detect the position of the fiducial markers on the MR images.

The headphones are able to expand and pivot to accommodate different head sizes. The multiple geometric variations make registration more challenging. Vendors may want to consider placing permanent fiducial markers on the headphones so they can be registered and added to the system hardware attenuation maps for correction.

We attempted to improve the modified $\mu$ map by varying the regions of interest on the headphone transmission data

TABLE 2

Percentage Difference in Counts in Selected Human Brain Regions

\begin{tabular}{lcc}
\hline Structure or region & $\begin{array}{c}\text { Difference } \\
\text { before correction }\end{array}$ & $\begin{array}{c}\text { Difference } \\
\text { after correction }\end{array}$ \\
\hline Cerebellar-cortical & $-14.4 \%$ & $0.2 \%$ \\
\hline Inferior temporal & $-14.0 \%$ & $2.1 \%$ \\
\hline Temporal pole & $-12.6 \%$ & $2.2 \%$ \\
\hline Fusiform & $-11.5 \%$ & $1.6 \%$ \\
Superior temporal & $-7.8 \%$ & $2.8 \%$ \\
\hline Medial orbitofrontal & $-6.7 \%$ & $-0.2 \%$ \\
Caudate & $-3.6 \%$ & $2.2 \%$ \\
Rostral anterior & $-2.4 \%$ & $3.0 \%$ \\
$\quad$ cingulate & & \\
\hline Superior frontal & $0.3 \%$ & $3.9 \%$ \\
\hline Cuneus & $0.3 \%$ & $2.9 \%$ \\
\hline Inferior parietal & $0.9 \%$ & $4.2 \%$ \\
\hline Precuneus & $1.0 \%$ & $4.0 \%$ \\
\hline
\end{tabular}




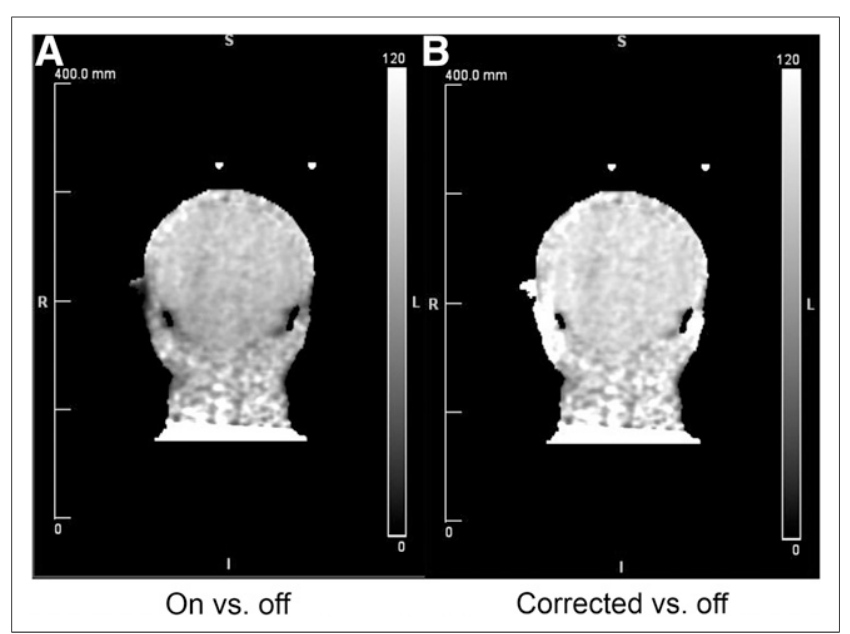

FIGURE 6. (A) Coronal image showing ratio of measured activity concentration from PET scan acquired with headphones over scan without headphones. (B) Coronal image showing ratio of activity concentration of patient PET scan with headphone attenuation correction over scan without headphones. A color version of this figure is available as a supplemental file at http:// tech.snmjournals.org.

to include more or less of the headphones in the region. We suspected that including more of the firm outer shell would yield higher correction values as long as air outside the headphones was not included. Modifying the regions to include either more or less of the headphones did not yield substantially different correction values. We were able to eliminate most of the attenuation effect of the headphones. We acquired a UTE sequence in an attempt to localize the headphones with an MR acquisition. If successful, this step would eliminate the need for fiducial markers for image registration. We were able to see the foam padding of the headphones with the UTE sequence; however, the image contrast from the UTE sequence was not enough to use for accurate image registration. Because the headphones were not visible with the UTE sequence, it did not provide an advantage over the Dixon sequence-based attenuation correction for the headphones and was not used for attenuation correction in this study. All attenuation correction was performed using the Dixon-based $\mu$ map in combination with the previously measured attenuation map of the headphones.

Substantial underestimation of activity was observed on the human subject, especially in regions near the headphones. Quantitative PET/MR studies of the brain will benefit from using attenuation maps that include attenuation from the headphones. We observed a slight overestimation of regional intensities in several areas of the human brain. Part of the reason for the observed overestimation may be the small change in tracer distribution due to the time difference between the emission scans with and without the headphones.

Future studies will evaluate the effect of headphone attenuation on quantitative imaging with other PET tracers. We will also further improve the headphone attenuation correction workflow to apply the method on a larger population.

\section{CONCLUSION}

MR-compatible headphones are a source of attenuation in PET/MR studies. Headphones create up to a $13.2 \%$ decrease in activity concentration in phantom studies. Similar underestimation was observed on a human subject. A practical approach to including the headphones in the attenuation maps was proposed and was shown to accurately correct for the headphone attenuation effects in both phantom and human PET/MR studies. Our study indicated that the attenuation effects of the headphones should be considered and corrected for quantitative neurologic PET/MR studies.

\section{DISCLOSURE}

No potential conflict of interest relevant to this article was reported.

\section{ACKNOWLEDGMENTS}

We thank Michael Harrod and Linda Becker at Washington University for their technical expertise and help with acquisitions.

\section{REFERENCES}

1. Carney JP, Townsend DW, Rappoport V, Bendriem B. Method for transforming CT images for attenuation correction in PET/CT imaging. Med Phys. 2006;33: 976-983.

2. Coombs BD, Szumowski J, Coshow W. Two-point Dixon technique for water-fat signal decomposition with B0 inhomogeneity correction. Mag Reson Med. 1997; 38:884-889.

3. Martinez-Möller A, Souvatzoglou M, Delso G, et al. Tissue classification as a potential approach for attenuation correction in whole-body PET/MRI: evaluation with PET/CT data. J Nucl Med. 2009;50:520-526.

4. Reichert IL, Robson MD, Gatehouse PD, et al. Magnetic resonance imaging of cortical bone with ultrashort TE pulse sequences. Magn Reson Imaging. 2005;23:611-618.

5. Keereman V, Fierens Y, Broux T, De Deene Y, Lonneux M, Vandenberghe S. MRI-based attenuation correction for PET/MRI using ultrashort echo time sequences. J Nucl Med. 2010;51:812-818.

6. Navalpakkam BK, Braun H, Kuwert T, Quick HH. Magnetic resonance-based attenuation correction for PET/MR hybrid imaging using continuous valued attenuation maps. Invest Radiol. 2013;48:323-332.

7. Berker Y, Franke J, Salomon A, et al. MRI-based attenuation correction for hybrid PET/MRI systems: a 4-class tissue segmentation technique using a combined ultrashort-echo-time/Dixon MRI sequence. J Nucl Med. 2012;53:796804.

8. Mantlik F, Hofmann M, Werner MK, et al. The effect of patient positioning aids on PET quantification in PET/MR imaging. Eur J Nucl Med Mol Imaging. 2011;38:920-929.

9. Delso G, Martinez-Moller A, Bundschuh RA, et al. Evaluation of the attenuation properties of MR equipment for its use in a whole-body PET/MR scanner. Phys Med Biol. 2010;55:4361-4374.

10. Kartmann R, Paulus DH, Braun H, et al. Integrated PET/MR imaging: automatic attenuation correction of flexible RF coils. Med Phys. 2013;40:082301.

11. MacDonald LR, Kohlmyer S, Liu C, Lewellen TK, Kinahan PE. Effects of MR surface coils on PET quantification. Med Phys. 2011;38:2948-2956.

12. Paulus DH, Braun H, Aklan B, Quick HH. Simultaneous PET/MR imaging: MRbased attenuation correction of local radiofrequency surface coils. Med Phys. 2012;39:4306-4315.

13. Tellmann L, Quick HH, Bockisch A, Herzog H, Beyer T. The effect of MR surface coils on PET quantification in whole-body PET/MR: results from a pseudo-PET/MR phantom study. Med Phys. 2011;38:2795-2805.

14. Son YD, Kim HK, Kim ST, Kim NB, Kim YB, Cho ZH. Analysis of biased PET images caused by inaccurate attenuation coefficients. J Nucl Med. 2010;51: $753-760$. 